\title{
Biologic and Targeted Synthetic DMARD Utilization in the United States: Adelphi Real World Disease Specific Programme for Rheumatoid Arthritis
}

\author{
Elizabeth A. Holdsworth • Bethany Donaghy · Kathleen M. Fox • \\ Pooja Desai (D) - David H. Collier · Daniel E. Furst
}

Received: July 2, 2021 / Accepted: August 3, 2021 / Published online: September 2, 2021

(C) The Author(s) 2021

\begin{abstract}
Introduction: In patients with inadequate response or intolerance to first biologic diseasemodifying antirheumatic drug (bDMARD), guidelines recommend switching to an agent of different mechanism of action or to another bDMARD. However, the reasons behind switching between bDMARD/targeted synthetic (ts)DMARD are not well documented in many studies. The objective of this study was to assess the rheumatologists' perceptions and behaviors towards choice of initial $\mathrm{b} /$ tsDMARD treatment and reasons for switching between bDMARDs/tsDMARDs, in the context of present treatment patterns.
\end{abstract}

Supplementary Information The online version contains supplementary material available at https:// doi.org/10.1007/s40744-021-00357-1.

E. A. Holdsworth · B. Donaghy

Adelphi Real World, Bollington, UK

K. M. Fox $(\bowtie)$ · P. Desai · D. H. Collier

Amgen Inc., Thousand Oaks, CA 91320, USA

e-mail: kfox02@amgen.com

D. E. Furst

Division of Rheumatology, David Geffen School of Medicine, University of California, Los Angeles, CA, USA

D. E. Furst

University of Washington, Seattle, WA, USA

D. E. Furst

University of Florence, Florence, Italy
Methods: This was a retrospective analysis of data collected from the 12th Adelphi Real World Disease Specific Programme for rheumatoid arthritis (RA). Qualified rheumatologists involved in treatment decision-making for $\geq 10$ patients a month completed patient record forms (PRFs). Patients aged $\geq 18$ years with RA diagnosis and receiving bDMARD/tsDMARD were included. The outcomes assessed were proportion of patients receiving bDMARD/tsDMARD at molecule and class levels; rheumatologist-reported reasons for choice of therapy; proportion of patients who switched bDMARDs/tsDMARDs; and rheumatologist-reported reasons for switching therapies.

Results: Eighty-six rheumatologists completed PRFs for 1027 patients. Of these, 621 were receiving bDMARD/tsDMARD at data collection. The majority (73\%) of patients received first-line bDMARD/tsDMARD, and at first-line, $68 \%$ received a tumor necrosis factor inhibitor (TNFi) and 21\% received a Janus kinase inhibitor (JAKi). The response option of strong overall efficacy was the primary reason for selecting first-line and second-line bDMARD/ tsDMARD. A total of 163 patients had switched from first-line $b /$ tsDMARD to second-line b/tsDMARD therapy. Of these, 44,28 , and $17 \%$ had switched from TNFi to another TNFi, TNFi to non-TNF biologic, and TNFi to JAKi, respectively. Lack of efficacy and worsening disease were the most frequent reasons for switching therapies. 
Conclusions: TNFis remain the most prescribed $\mathrm{b} /$ tsDMARD for first-line and second-line treatments. Strong overall efficacy was the primary reason for selecting therapy and loss of efficacy was the primary reason for switching therapy.

Keywords: Biologic DMARDs; Rheumatoid arthritis; Tumor necrosis factor inhibitors; Targeted synthetic DMARDs

\section{Key Summary Points}

\section{Why carry out this study?}

Limited studies have evaluated the rheumatologist's perceptions towards choice of $b /$ tsDMARD therapy and reasons behind switching between $\mathrm{b} /$ tsDMARDs in real-world settings.

The aim of this study was to identify the rheumatologist-reported reasons for choice of b/tsDMARD and reasons for switching in patients with rheumatoid arthritis receiving bDMARDs/tsDMARDs.

\section{What was learned from the study?}

Tumor necrosis factor inhibitors (TNFis) were the most prescribed agents at firstline, and nearly half of the patients who switched $b / t s D M A R D s$ received TNFi as a second-line therapy rather than a nonTNF biologic or a Janus kinase inhibitor (JAKi).

Rheumatologists followed the ACR 2015 and EULAR 2019 guidelines by switching to a different biologic with similar mechanism of action upon treatment failure with the initial b/tsDMARD; however, the ACR 2021 guidelines recommend switching to a biologic with a different mechanism of action.

Based on this study, rheumatologists' decision-making for $b / t s D M A R D s$ remains largely driven by clinical factors, but healthcare policy and safety concerns are also considered.

\section{INTRODUCTION}

Rheumatoid arthritis (RA) is a chronic, progressive disease, which may negatively impact patients' ability to perform daily activities and reduces the health-related quality of life [1]. The disease affects approximately 1.4 million adults with an estimated prevalence of $0.5 \%$ in the United States (US) [2]. The economic burden associated with RA is substantial, with a 2010 estimate of US $\$ 19.3$ billion in direct and indirect costs, and with an additional US \$19.9 billion resulting from the deterioration of quality of life and premature mortality [3]; these costs may have escalated in the past decade.

Advancements in elucidating the molecular pathways underlying the disease have led to the development of new therapies, including biologic disease-modifying antirheumatic drugs (bDMARDs) and targeted synthetic DMARDs (tsDMARDs), which have revolutionized the treatment landscape over the last two decades. The various therapeutic options available for managing the disease include glucocorticoids and DMARDs including conventional synthetic DMARDs (methotrexate, sulfasalazine, and leflunomide), bDMARDs (tumor necrosis factor inhibitors [TNFis]: etanercept, adalimumab, infliximab, certolizumab pegol, and golimumab and non-TNF biologics: tocilizumab, sarilumab, abatacept, anakinra, and rituximab), and tsDMARDs (Janus kinase inhibitors [JAKis]: tofacitinib, baricitinib, and upadacitinib) [4]. The primary goal of treatment is to reduce the signs and symptoms of the disease, prevent structural damage to bone and cartilage, and normalize physical function and social participation, thereby improving the health-related quality of life [4]. The American College of Rheumatology (ACR) 2015 and the European League Against Rheumatism (EULAR) 2019 guidelines recommend methotrexate as a firstline treatment strategy in patients with early RA, and methotrexate in combination with bDMARDs or tsDMARDs in patients with moderate or high disease activity and poor prognosis. These guidelines also recommend switching the bDMARD/tsDMARD to a molecule with a different mechanism of action (MoA, i.e., other 
non-TNF biologic or tsDMARD [JAKi]) or to a second TNFi upon inadequate response to or failure of prior therapy $[1,4]$.

Given the increasing availability of a wide host of treatment options to manage the condition, the increasing body of evidence of treatment effectiveness, and the ACR 2021 guidelines, clearer understanding of real-world clinical practices is warranted. Further, physicians' preferences towards choosing $\mathrm{b} / \mathrm{tsDMARD}$ therapies, as well as reasons for switching between the classes of bDMARDs/ tsDMARDs should be reviewed to understand the current clinical decision-making for patients with RA. In particular, the treatment sequences and reasons for switching have not been investigated in patients receiving bDMARD/ tsDMARD in real-world settings. Therefore, this cross-sectional survey was utilized to assess, in the context of present treatment patterns, the rheumatologists' perception around the initial choice of $b /$ tsDMARD, and reasons for switching therapies in patients receiving bDMARD/ tsDMARD.

\section{METHODS}

\section{Study Design}

This was a secondary data analysis study, which utilized data collected between December 2019 and September 2020 from the 12th Adelphi Real World Disease Specific Programme (DSP) for RA-a non-interventional, observational, crosssectional survey dataset collected using a combination of rheumatologist surveys and rheumatologist-completed patient record forms (PRFs).

\section{Rheumatologist's and Patient's Eligibility Criteria}

Rheumatologists (MD or DO board certified in rheumatology) actively managing patients with RA were identified from publicly available registers of healthcare professionals across the US. Those qualified with $\geq 3$ years of clinical practice experience and involved in treatment decision-making for a minimum of ten RA patients per calendar month were eligible. Rheumatologists were invited to participate in an online cross-sectional survey, which captured their perceptions towards the treatment and management of RA. Following completion of the online survey, each rheumatologist completed a workload survey, which documented overall RA workload over a 5-day period and collected data on the total number of patients seen. Rheumatologists also completed a detailed PRF for the next 12 consecutive patients with RA who consulted in their clinic, regardless of patient disease severity and current treatments. The survey captured data pertaining to patient demographics, current and prior treatments, and clinical outcomes. The detailed methodology and the steps involved in conducting the DSP survey were described previously [5].

Patients aged $\geq 18$ years with rheumatologist-confirmed RA diagnosis and those receiving a bDMARD/tsDMARD at the time of data collection were included. Those $<18$ years, with active participation in any ongoing clinical trial, and with comorbidities including psoriasis, psoriatic arthritis, ankylosing spondylitis, non-radiographic axial spondyloarthritis, lupus erythematosus, Crohn's disease, ulcerative colitis, or with any malignancy (including leukemia and lymphoma) or metastatic solid tumor were excluded. Ethical committee approval was waived by the Western Institutional Review Board for data collection and analysis as this study was based on a retrospective analysis of an existing dataset. Patient anonymity and confidentiality were safeguarded in compliance with the Health Insurance Portability and Accountability Act. Rheumatologists were compensated by Adelphi Real World for their time to complete the survey and study-related tasks.

\section{Outcome Measures}

The outcomes of this survey were to examine the reasons rheumatologists reported for $\mathrm{b} / \mathrm{tsDMARD}$ treatment choice; to assess the proportion of patients who switched between bDMARD/tsDMARD treatments at the molecule 
and class level; to describe rheumatologist-reported reasons for switching therapy; and to describe the proportion of patients prescribed a bDMARD/tsDMARD at molecule and class levels among those administered with bDMARDs/ tsDMARDs. The response options for reason for choice of therapy were grouped as follows: (1) clinical reasons (strong overall efficacy; inhibition of disease progression; reduction in stiffness; familiarity/experience with product; maintenance of efficacy over time; strong efficacy as monotherapy; convincing efficacy data in clinical trials; achievement of treat-to-target (T2T) goal; achievement of low disease activity (LDA); achievement of clinical remission; control of acute episode/flare; fast onset of action; and allows reduction in steroid use), (2) patientcentric reasons (enabling patient to perform everyday tasks/usual activities; sustained pain relief; improvement or maintenance of quality of life [work, leisure, outlook, etc.]; reduction of fatigue; low out-of-pocket cost/affordability for patients; improving patient's mood/state of mind), (3) contraindication/safety (suitability for patients with cardiovascular risk; does not exacerbate comorbidities; does not exacerbate non-autoimmune conditions; improves concomitant autoimmune conditions; overall safety profile; drug-drug interaction data, and contraindication data), (4) healthcare system (product provides a reasonable cost-benefit ratio, product inclusion in local/national formulary, and few administrative controls on product use), and (5) therapy administration (acceptability of mode of delivery for the patient and ease of product use). Further, subgroup analysis based on the number of years a rheumatologist held their qualification (board certified: before 1983-1995, 1996-2005, and 2006-after 2016) was also performed to observe the changing trend in prescribing patterns and choice of therapy.

\section{Statistical Analysis}

Descriptive statistics were used for presenting the results of this analysis. Categorical variables were presented as frequency and percentage. Analyses were performed in the overall population and by line of therapy (i.e., first-line [patients who were receiving their first bDMARD/tsDMARD therapy at data collection] and second-line [patients who were receiving their second bDMARD/tsDMARD therapy at data collection]). All analyses were performed using Stata v15.0 or later [6].

\section{RESULTS}

\section{Participant Characteristics}

Eighty-six rheumatologists participated in the cross-sectional survey. The majority were male (69\%) and each rheumatologist managed an average of 87.2 patients with RA per month. Over $80 \%$ worked in private office settings and more than half $(53 \%)$ were involved in clinical trials either previously or at the time of data collection. The proportions of rheumatologists who were board certified between 1983 and 1995, 1996 and 2005, and 2006 and 2016 were 33,30 , and 24\%, respectively. Each rheumatologist completed the PRFs for approximately 12 patients with RA accounting for a total of 1027 PRFs. Of these, 32 were excluded due to comorbid inflammatory conditions. Of the remaining 995 PRFs, 621 (60.5\%) were included in this analysis following exclusion of 374 patients who did not receive an advanced therapy (bDMARD/tsDMARD) at the time of data collection.

\section{Proportion of Patients on Current Advanced Therapy (bDMARD or tsDMARD)}

The majority (73\%) of patients were receiving $\mathrm{b} /$ tsDMARD first-line, $15 \%$ second-line, and $11 \%$ third-line and subsequent-line advanced therapy. Adalimumab, etanercept, and tofacitinib were the most common first-line $\mathrm{b} / \mathrm{tsDMARD}$ received by 26,24 , and $17 \%$ of patients, respectively. Abatacept was the most common second-line b/tsDMARD received by $28 \%$ of patients, followed by etanercept and tofacitinib with $14 \%$ each. Tocilizumab was the most common third-line and subsequent-line 
b/tsDMARD received by $20 \%$ of patients (Fig. 1a). The only marketed biosimilars in the US were for infliximab: Renflexis and Inflectra, received by $<1 \%$ of patients at first-line. Most patients received TNFis as first-line $b / t s D M A R D$
(68\%), roughly equal proportions of patients used TNFis and "other" biologics as second-line therapy (37 and 39\%, respectively), and "other" biologics were used most as third-line therapy $(48 \%)$. Approximately a quarter $(21-29 \%)$ of

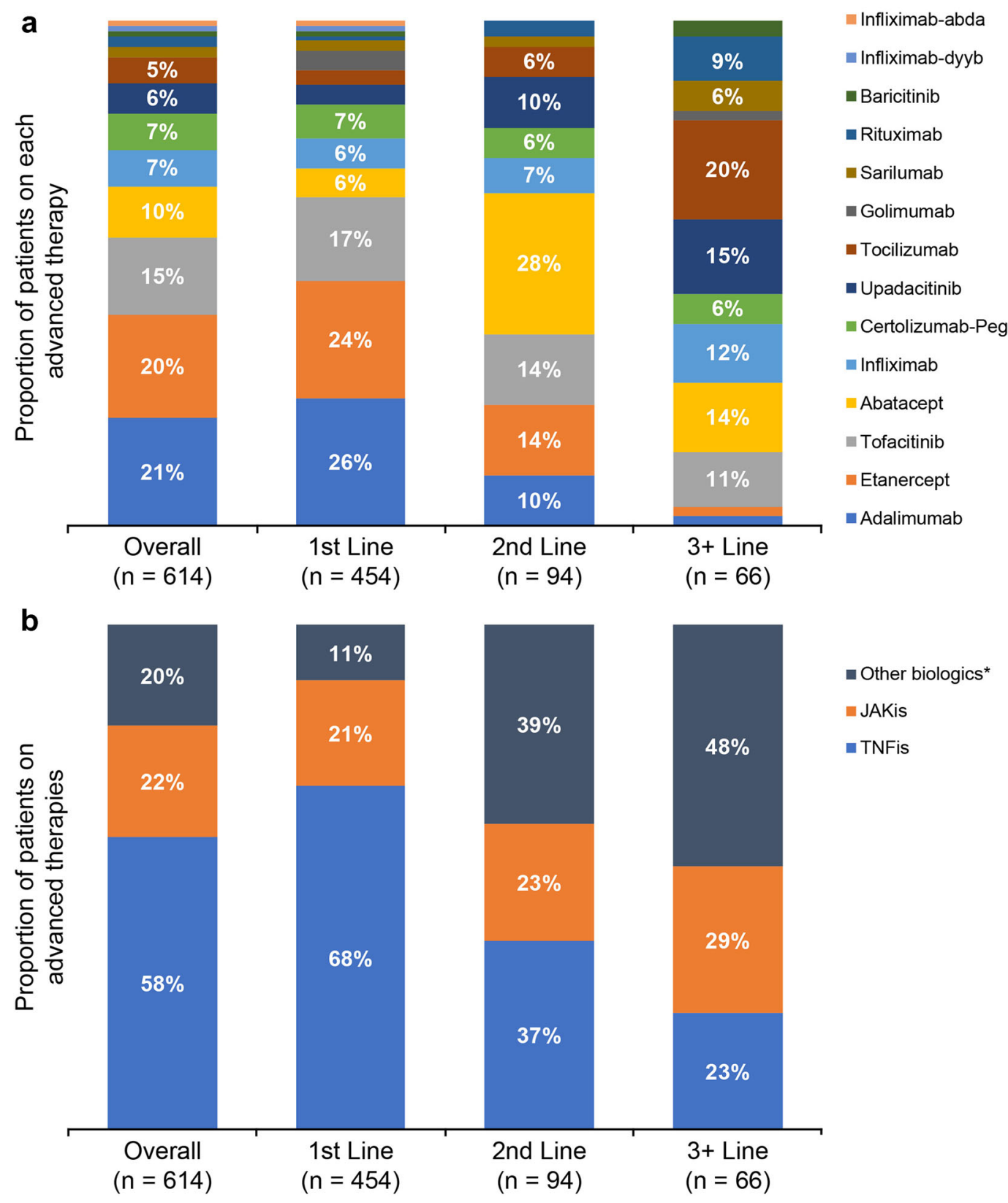

Fig. 1 Proportion of patients on advanced therapies by line of therapy at molecule level (a), and class level (b). ${ }^{*}$ Other biologics include non-TNF biologics such as IL-6 antagonists (tocilizumab and sarilumab), abatacept, and rituximab. Infliximab-dyyb and infliximab-abda were the biosimilars of originator biologic infliximab. $I L$ interleukin, $J A K i$ Janus kinase inhibitor, TNFi tumor necrosis factor inhibitor 
patients were receiving JAKis across any line of b/tsDMARD (Fig. 1b).

\section{Rheumatologist-Reported Reasons for Choice of Therapy}

Clinical reasons (strong overall efficacy, inhibition of disease progression, reduction in joint stiffness, maintenance of efficacy over time, familiarity or experience with medical product, achievement of clinical remission or LDA, etc.) were the most common among the rheumatologist-reported reasons for choosing a bDMARD/ tsDMARD across all lines of therapy (reported by $97 \%$ of rheumatologists), followed by patient-centric reasons (enabling patient to perform everyday tasks/usual activities, sustained pain relief, improvement or maintenance of quality of life, reduction of fatigue, and affordability) with $68-76 \%$ of rheumatologists reporting this as the second common reason for choosing a bDMARD/tsDMARD therapy, and then contraindication/safety reasons (suitability for patients with cardiovascular risk; does not exacerbate comorbidities; does not exacerbate non-autoimmune conditions; improves concomitant autoimmune conditions; overall safety profile; drug-drug interaction data, and contraindication data) reported by $53-63 \%$ of rheumatologists across all lines of therapy (Fig. 2a). Strong overall efficacy and inhibition of disease progression were the key reasons provided by rheumatologists for choosing a bDMARD/tsDMARD across all lines of therapy. Similar reasons (clinical reasons [strong overall efficacy and inhibition of disease progression], patient-centric reasons, and contraindication/ safety reasons) - in that order-were reported as the most common reasons for making a firstline treatment choice across all b/tsDMARD classes including TNFis, non-TNF biologics, and JAKis (Fig. 2b). When considering a second line of therapy (Fig. 2c), although the number of patients were fewer and may need to be considered conservatively-again, clinical reasons, patient-centric reasons, and contraindication/ safety reasons, in that order, were the most common reasons for the current use of TNFis, non-TNF biologics, and JAKis, although some variability in the proportions was observed. Similar to the first-line b/tsDMARD choice, strong overall efficacy was the most common reason provided by rheumatologists for choosing across all three classes (TNFis, non-TNF biologics, and JAKis) for second-line b/tsDMARDs. Throughout, healthcare policies and convenience of administration were reported as reasons but with much lower frequency. It was noted that administration was more frequently a selected response than healthcare policy when it came to the JAKi or second-line and third-line and subsequent-line b/tsDMARDs, although nearly always in much lower frequency than the others above (Fig. 2c).

Subgroup analysis based on the number of years since board certification in rheumatology showed strong overall efficacy, inhibition of disease progression, reduction in joint stiffness, familiarity or experience with treatment agent, and enabling patient to perform daily activities as the most common reasons for making a firstline $\mathrm{b} / \mathrm{tsDMARD}$ choice across all board certification years. For second-line b/tsDMARD choice, maintenance of efficacy over time became one of the key reasons along with strong overall efficacy, inhibition of disease progression, reduction in joint stiffness, and familiarity or experience with treatment agent across all board certification years (Fig. S1; Supplementary Material).

\section{Proportion of Patients Switching Between Advanced Therapies}

A total of 163 patients $(26.2 \%$; 163/621) had previously switched from first-line to secondline b/tsDMARD and 68 of them $(42 \% ; 68 / 163)$ had switched from second-line to third-line $\mathrm{b} / \mathrm{tsDMARD}$. Of the 163 patients, five were excluded as the rheumatologists were unable to specify switch details due to missing data. Of the remaining 158 patients who had switched from first-line to second-line b/tsDMARD, 122 $(77 \% ; 122 / 158)$ had switched from first-line bDMARD to second-line bDMARD, 30 (19\%; 30/158) had switched from first-line bDMARD to second-line tsDMARD, and six $(4 \% ; 6 / 158)$ had switched from first-line tsDMARD to 


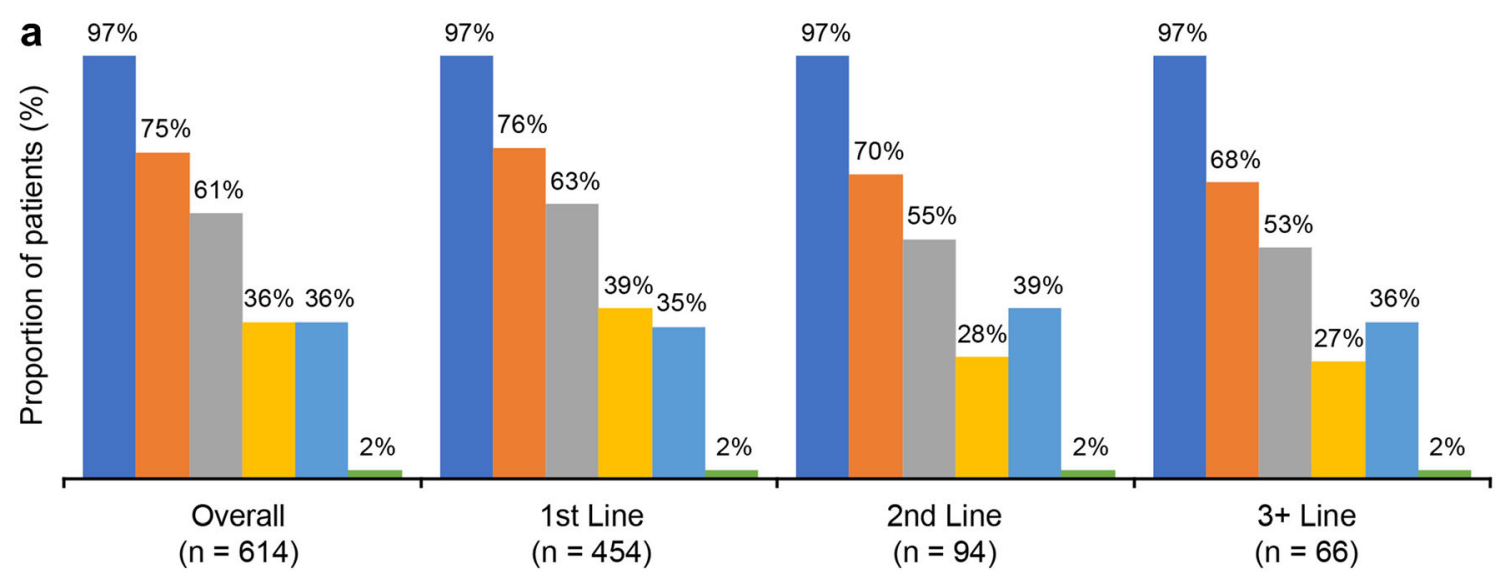

b $97 \%$

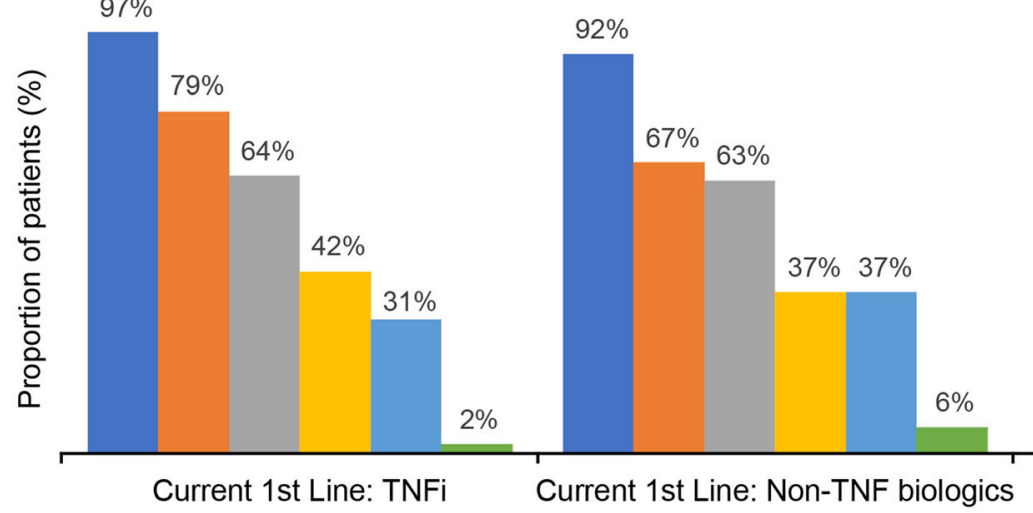
$(n=307)$ $(n=51)$

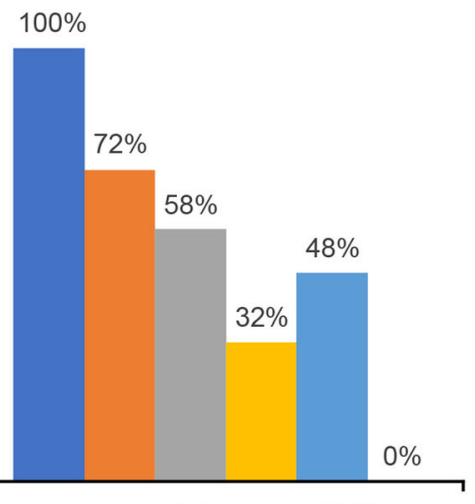

Current 1st Line: JAKi

C $100 \%$

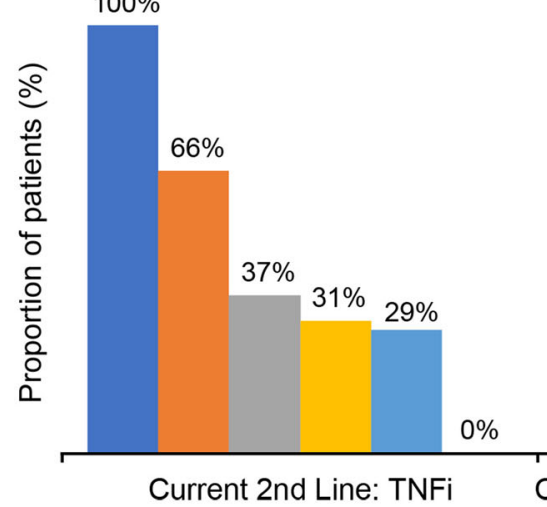
$(\mathrm{n}=35)$

$95 \%$

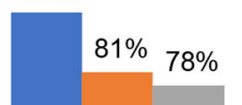

$(n=96)$ 
second-line bDMARD. Of the 122 patients who had switched from first-line bDMARD to second-line bDMARD, $57.4 \%$ (70/122) switched from TNFi to another TNFi, 36.1\% (44/122) switched from TNFi to non-TNF biologic, 3.3\% (4/122) switched from non-TNF biologic to another non-TNF biologic, and 3.3\% (4/122) switched from non-TNF biologic to TNFi. Of the 30 patients who had switched from first-line bDMARD to second-line tsDMARD, 90\% (27/30) and 10\% (3/30) switched from TNFi to JAKi and from non-TNF biologic to JAKi, respectively (Fig. 3).

Sixty-eight patients had switched from second-line to third-line b/tsDMARD in this study. Of these, $44(64.7 \% ; 44 / 68)$ patients switched from second-line bDMARD to third-line
bDMARD, 16 (23.5\%; 16/68) patients switched from second-line bDMARD to third-line tsDMARD, and eight (11.8\%; 8/68) switched from second-line tsDMARD to third-line bDMARD. Of the 44 patients who switched between bDMARDs, $36.4 \% \quad(16 / 44)$ switched from TNFi to another TNFi, 38.6\% (17/44) switched from TNFi to non-TNF biologic, $20.4 \%$ (9/44) switched from non-TNF biologic to another non-TNF biologic, and 4.5\% (2/44) switched from non-TNF biologic to TNFi. Of the 16 patients who switched from second-line bDMARD to third-line tsDMARD, 87.5\% (14/16) and $12.5 \%(2 / 16)$ switched from TNFi to JAKi and from non-TNF biologic to JAKi, respectively (Fig. 3).

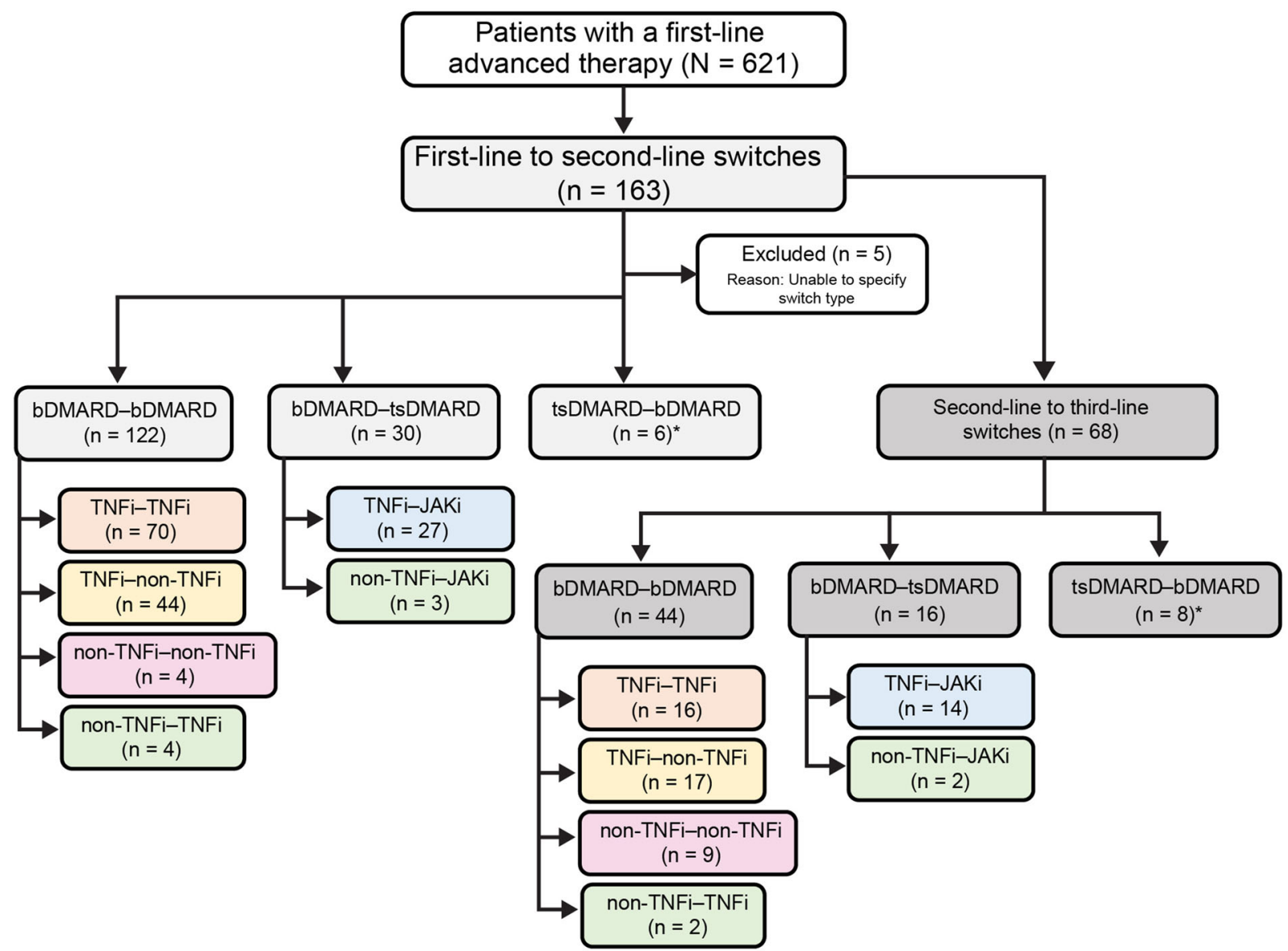

Fig. 3 Proportion of patients with RA switching between advanced therapies. ${ }^{*}$ Analyzed patient number was very low. $B D M A R D$ biologic disease-modifying antirheumatic drug, JAKi Janus kinase inhibitor, TNFi tumor necrosis factor inhibitor, tsDMARD targeted synthetic diseasemodifying antirheumatic drug 


\section{Reasons for Switching Between Advanced Therapies}

Secondary loss of efficacy (loss of response over time) was the primary reason for switching from first-line bDMARD to second-line bDMARD (55\%) and from first-line bDMARD to secondline tsDMARD (44\%). The same reasons were reported in over half of the patients for switching from second-line bDMARD to thirdline bDMARD (54\%) and from second-line bDMARD to third-line tsDMARD (53\%). Across all switch types including switch from first-line to second-line and second-line to third-line $\mathrm{b} / \mathrm{tsDMARD}$, less than $20 \%$ of patients switched therapy due to safety or tolerability reasons (data not shown).

Secondary inefficacy or loss of therapeutic response over time followed by worsening disease were the main reasons for switching from first-line TNFi to second-line TNFi, first-line TNFi to second-line non-TNF biologic, and firstline TNFi to second-line JAKi. The same reasons were reported by rheumatologists for switching from second-line to third-line $b$ /tsDMARDs (Fig. 4).

\section{DISCUSSION}

This analysis of real-world data from practicing rheumatologists and their patients with RA receiving bDMARD/tsDMARD therapies showed that TNFis (adalimumab and etanercept) were the most commonly used biologics at first line; these were commonly followed by another TNFi, if a switch in treatment was required. Furthermore, clinical reasons (strong overall efficacy; inhibition of disease progression; reduction in stiffness; familiarity/experience with product; maintenance of efficacy over time, etc.) and patient-centric reasons (enabling patient to perform everyday tasks/usual activities; sustained pain relief; improvement or maintenance of quality of life; reduction of fatigue; low out-of-pocket cost/affordability for patients; improving patient's mood/state of mind) were the most common rheumatologistreported reasons for choosing a bDMARD/

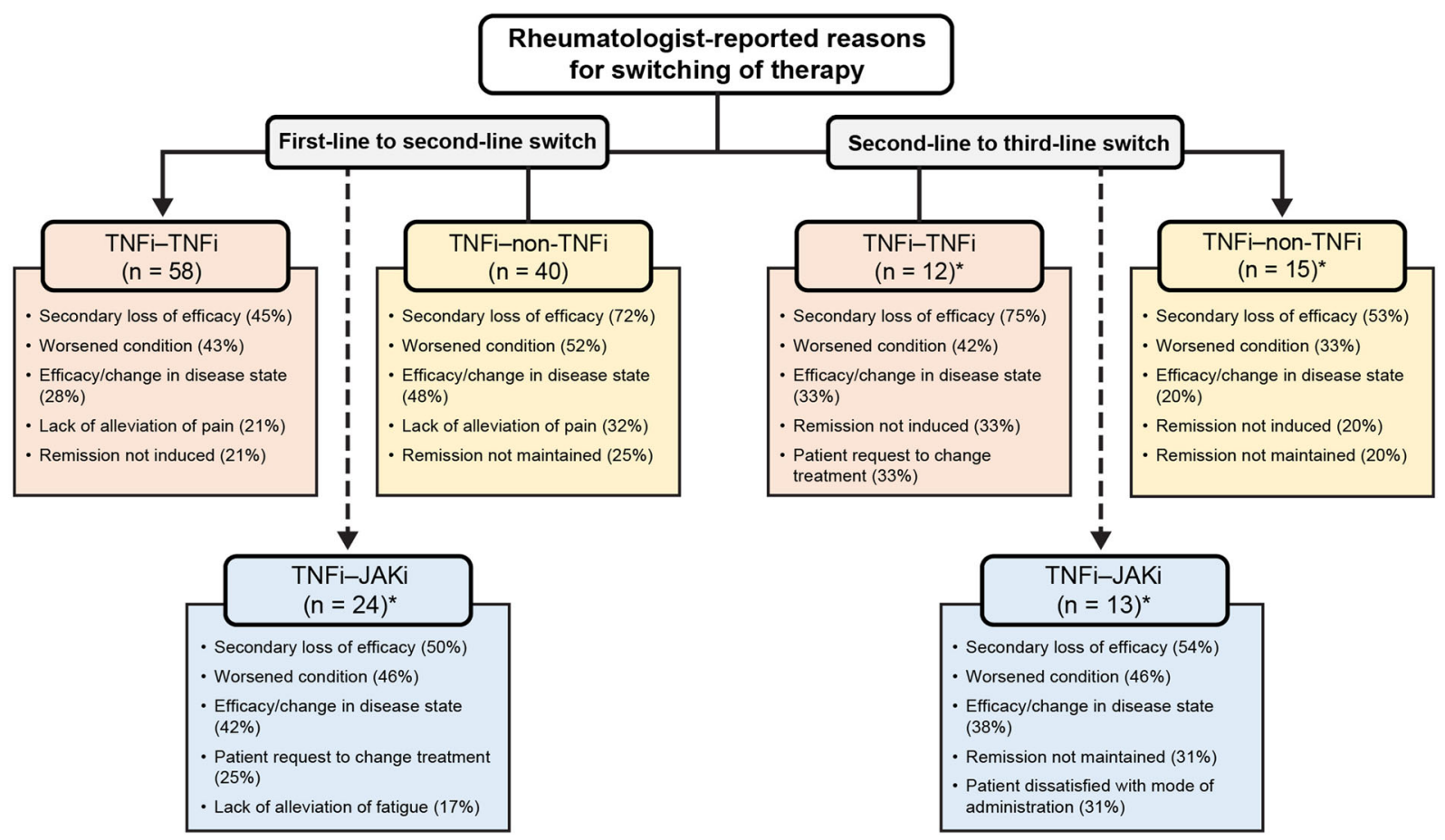

Fig. 4 Rheumatologist-reported reasons for switching between advanced therapies. ${ }^{*}$ Analyzed patient number was very low. $J A K i$ Janus kinase inhibitor, TNFi tumor necrosis factor inhibitor 
tsDMARD across all lines of therapy. These results align with the current perspective that T2T strategies are the cornerstone to achieve remission or LDA in RA [7]. Healthcare policies and convenience of administration were reported with lower frequency by rheumatologists to be further reasons for choosing a bDMARD/ tsDMARD across all lines of therapy in the overall population. Convenience of administration had some role to play in second-line and third-line b/tsDMARDs compared with healthcare policies; this makes intuitive sense when considering "back-up" treatments. Moreover, secondary inefficacy (loss of response over time) was the primary reason for switching a b/tsDMARD, both within bDMARDs and for switching between bDMARDs and tsDMARDs.

The ACR 2015 and EULAR 2019 guidelines (guidelines available at the time of conduct of this study) recommend treatment with another bDMARD or a tsDMARD in case of treatment failure with initial bDMARD/tsDMARD. The guidelines also recommend treatment with an agent of another treatment class (different MoA) or a second TNFi upon failure of the first TNFi therapy [1, 4]. However, the ACR 2021 guideline (published after the conduct of this study) recommends switching to an agent of different MoA (i.e., non-TNF biologic or JAKi), and no longer recommends switching to a second TNFi upon insufficient response to prior TNFi therapy [8]; a conditional recommendation with low-quality evidence. Previous studies that examined the prescribing patterns, treatment practices, and persistence among patients with RA in the US, Europe, and Japan, reported that most patients were prescribed a TNFi as their first-line targeted therapy and approximately half of these patients cycled to another TNFi as the second therapy [9-12]. This speaks to a nuanced response, as some studies indicate that switching to a second TNFi remains effective if the patient has initial response but loses it over time or if there is an adverse event to the first TNFi [13]. Also, a recent literature review concluded that treatment strategies (T2T) may be more important than a specific drug to control RA [7]. In our study, we found that $44 \%$ of patients switched from TNFi to another TNFi, $28 \%$ switched from TNFi to non-TNF biologic, and $17 \%$ switched from TNFi to JAKi as the firstline to second-line switch in accordance with the previous guideline recommendations $[1,4]$ and as observed in earlier studies [9-12]. We also found that rheumatologists mainly rely on clinical and safety evidence (clinical, patientcentric, and safety reasons), and the influence of healthcare policies or formularies in their decision-making was relatively less compared with clinical factors, consistent with other studies [14]. The clinical needs of their patients appear to be the primary drivers of clinical decisionmaking. Contraindication/safety was only the third most important reason for choice of therapy, after clinical and patient-centric reasons. The primary reason given by rheumatologists for making a first-line (TNFi, non-TNF biologic, and JAKi) and second-line (TNFi, nonTNF biologic, and JAKi) treatment choice was strong overall efficacy. This is supported by a literature review focusing on strategies for attaining RA remission which concluded that switching to a second TNFi may be beneficial after the discontinuation of the first, in spite of the reason for discontinuation [15]. A similar reason was reported as the key reason to prescribe the first-line targeted therapy in the previous studies based on Adelphi DSP [10, 11]. Analysis of data from the Corrona RA registry showed that approximately one-third and half of the patients who initiated a bDMARD discontinued their treatment within 12 and 24 months of initiation, respectively. Loss of efficacy $(35.8 \%)$ followed by physician preference $(27.8 \%)$, safety $(20.1 \%)$, patient preference (17.9\%), and no access to treatment $(9.0 \%)$ were the reasons for discontinuation or switching of bDMARDs [16]. Another study based on the Veteran Affairs data collected between 1999 and 2007 showed that nearly $50 \%$ of patients had either stopped or switched their biologic agent, with the most common reasons reported as adverse events (in 48\%) and inefficacy (in 43\%), and the factors significantly associated with stopping or switching were higher Disease Activity Score (DAS28) and Health Assessment Questionnaire scores [17]. Similar reasons (lack of efficacy, safety, patient preference, and disease remission) were reported in other realworld observational studies that examined the 
physician-reported reasons for discontinuation or switching of therapies in RA [18-26]. In our study, we found that secondary loss of efficacy was the main reason for switching $b / t s D M A R D s$, which was in accordance with the previous observational studies [10, 11, 24].

\section{Limitations}

Some limitations need to be considered when interpreting the results of this analysis. First, rheumatologist selection bias was possible as rheumatologist participation was based on a willingness to take part in the survey. Second, the patient sample collected was not a randomized sample, as the DSP is based on a consecutive sampling strategy. Therefore, it is representative of the consulting patient population, and may over-represent patients who consult more frequently. Third, the quality of data depends to a large extent on the accurate reporting of information by rheumatologists; however, the data used in this study were based on medical record data extraction at the time of the consultation. Therefore, it is unlikely to be subject to recall bias.

\section{CONCLUSIONS}

TNFis were the most prescribed first-line b/tsDMARDs, and among TNFis, adalimumab and etanercept were most prescribed in patients with RA. TNFi cycling (i.e., switching to another TNFi upon insufficient response to a first-line TNFi) was more common in current clinical practice and the proportion of patients who switched to a molecule of different MoA (nonTNF biologic/JAKi), per the recent ACR guidelines, was relatively low in the real-world setting. Clinical and patient-centric reasons, specifically, strong overall efficacy and inhibition of disease progression were the main reasons identified for choosing a bDMARD/tsDMARD, while loss of efficacy and worsening disease were the main reasons for switching b/tsDMARDs. Contraindication/safety was less frequently reported as reasons for choice of therapy than clinical and patient-centric reasons but more frequently reported than convenience of administration or healthcare policy reasons.

\section{ACKNOWLEDGEMENTS}

Funding. This study and journal's rapid Service Fee were funded by Immunex, a wholly owned subsidiary of Amgen Inc., Thousand Oaks, CA, USA.

Medical Writing and Editorial Assistance. Julie Wang, DPM, of Amgen Inc., and Lakshmi Narendra Bodduluru, PhD, of Cactus Life Sciences (part of Cactus Communications) provided writing and editorial assistance, funded by Amgen Inc.

Authorship. All named authors meet the International Committee of Medical Journal Editors (ICMJE) criteria for authorship for this article, take responsibility for the integrity of the work as a whole, and have given their approval for this version to be published.

Author Contributions. Study conception and design: Daniel E. Furst, David H. Collier, Elizabeth A. Holdsworth, Kathleen M. Fox, and Pooja Desai; Acquisition of data: Bethany Donaghy and Elizabeth A. Holdsworth; Analysis and interpretation of data: Bethany Donaghy, Daniel E. Furst, David H. Collier, Elizabeth A. Holdsworth, Kathleen M. Fox, and Pooja Desai; Drafting the manuscript or revising it critically for important intellectual content: Bethany Donaghy, Daniel E. Furst, David H. Collier, Elizabeth A. Holdsworth, Kathleen M. Fox, and Pooja Desai; Final approval of the version of the manuscript to be published: Bethany Donaghy, Daniel E. Furst, David H. Collier, Elizabeth A. Holdsworth, Kathleen M. Fox, and Pooja Desai.

Disclosures. Kathleen M. Fox, Pooja Desai, and David H. Collier are employees and stockholders of Amgen Inc. Elizabeth A. Holdsworth and Bethany Donaghy are employees of Adelphi Real World. Daniel E. Furst has received grants and consultancy fees from Actelion, Amgen, Corbus, Emerald, Galapagos, GSK, Horizon, 
Kadmon, NIH, Novartis, Pfizer, and Sanofi Aventis (all less than $\$ 10,000$ each for consultancy fees).

Compliance with Ethics Guidelines. Ethical committee approval was waived by the Western Institutional Review Board for data collection and analysis as this study was based on a retrospective analysis of an existing dataset.

Data Availability. The datasets generated during and/or analyzed during the current study are available from the corresponding author on reasonable request. Qualified researchers may request data from Amgen clinical studies. Complete details are available at the following: https://wwwext.amgen.com/science/ clinical-trials/clinical-data-transparencypractices/clinical-trial-data-sharing-request/ Journals.

Open Access. This article is licensed under a Creative Commons Attribution-NonCommercial 4.0 International License, which permits any non-commercial use, sharing, adaptation, distribution and reproduction in any medium or format, as long as you give appropriate credit to the original author(s) and the source, provide a link to the Creative Commons licence, and indicate if changes were made. The images or other third party material in this article are included in the article's Creative Commons licence, unless indicated otherwise in a credit line to the material. If material is not included in the article's Creative Commons licence and your intended use is not permitted by statutory regulation or exceeds the permitted use, you will need to obtain permission directly from the copyright holder. To view a copy of this licence, visit http:// creativecommons.org/licenses/by-nc/4.0/.

\section{REFERENCES}

1. Singh JA, Saag KG, Bridges SL Jr, et al. 2015 American College of Rheumatology guideline for the treatment of rheumatoid arthritis. Arthritis Rheumatol. 2016;68(1):1-26.
2. Hunter TM, Boytsov NN, Zhang X, Schroeder K, Michaud K, Araujo AB. Prevalence of rheumatoid arthritis in the United States adult population in healthcare claims databases, 2004-2014. Rheumatol Int. 2017;37(9):1551-7.

3. Birnbaum H, Pike C, Kaufman R, Marynchenko M, Kidolezi Y, Cifaldi M. Societal cost of rheumatoid arthritis patients in the US. Curr Med Res Opin. 2010;26(1):77-90.

4. Smolen JS, Landewé RBM, Bijlsma JWJ, et al. EULAR recommendations for the management of rheumatoid arthritis with synthetic and biological disease-modifying antirheumatic drugs: 2019 update. Ann Rheum Dis. 2020;79(6):685-99.

5. Anderson P, Benford M, Harris N, Karavali M, Piercy J. Real-world physician and patient behaviour across countries: Disease-Specific Programmes - a means to understand. Curr Med Res Opin. 2008;24(11):3063-72.

6. StataCorp. . Stata statistical software: release 15 . College Station: StataCorp LLC; 2017.

7. Drosos AA, Pelechas E, Voulgari PV. Treatment strategies are more important than drugs in the management of rheumatoid arthritis. Clin Rheumatol. 2020;39(4):1363-8.

8. Fraenkel L, Bathon JM, England BR, et al. American College of Rheumatology guideline for the treatment of rheumatoid arthritis. Arthritis Rheumatol. 2021. https://doi.org/10.1002/art.41752.

9. Chastek B, Chen C-I, Proudfoot C, Shinde S, Kuznik A, Wei W. Treatment persistence and healthcare costs among patients with rheumatoid arthritis changing biologics in the USA. Adv Ther. 2017;34(11):2422-35.

10. Sullivan E, Kershaw J, Blackburn S, Choi J, Curtis JR, Boklage S. Biologic disease-modifying antirheumatic drug prescription patterns for rheumatoid arthritis among United States physicians. Rheumatol Ther. 2020;7(2):383-400.

11. Sullivan E, Kershaw J, Blackburn S, Mahajan P, Boklage SH. Biologic disease-modifying antirheumatic drug prescription patterns among rheumatologists in Europe and Japan. Rheumatol Ther. 2020;7(3):517-35.

12. Wei W, Knapp K, Wang L, et al. Treatment persistence and clinical outcomes of tumor necrosis factor inhibitor cycling or switching to a new mechanism of action therapy: real-world observational study of rheumatoid arthritis patients in the united states with prior tumor necrosis factor inhibitor therapy. Adv Ther. 2017;34(8):1936-52. 
13. Bombardieri S, Ruiz AA, Fardellone P, et al. Effectiveness of adalimumab for rheumatoid arthritis in patients with a history of TNF-antagonist therapy in clinical practice. Rheumatology. 2007;46(7): 1191-9.

14. Schumock GT, Walton SM, Park HY, et al. Factors that influence prescribing decisions. Ann Pharmacother. 2004;38(4):557-62.

15. Papagoras C, Voulgari PV, Drosos AA. Strategies after the failure of the first anti-tumor necrosis factor alpha agent in rheumatoid arthritis. Autoimmun Rev. 2010;9(8):574-82.

16. Strand V, Miller P, Williams SA, Saunders K, Grant $\mathrm{S}$, Kremer J. Discontinuation of biologic therapy in rheumatoid arthritis: analysis from the Corrona RA registry. Rheumatol Ther. 2017;4(2):489-502.

17. Oei HB, Hooker RS, Cipher DJ, Reimold A. High rates of stopping or switching biological medications in veterans with rheumatoid arthritis. Clin Exp Rheumatol. 2009;27(6):926-34.

18. Ebina K, Hashimoto M, Yamamoto W, et al. Drug tolerability and reasons for discontinuation of seven biologics in 4466 treatment courses of rheumatoid arthritis-the ANSWER cohort study. Arthritis Res Ther. 2019;21(1):91.

19. Ebina K, Hashimoto M, Yamamoto W, et al. Drug tolerability and reasons for discontinuation of seven biologics in elderly patients with rheumatoid arthritis -the ANSWER cohort study. PLoS One. 2019;14(5):e0216624.

20. Ebina K, Hirano T, Maeda Y, et al. Drug retention of 7 biologics and tofacitinib in biologics-naïve and biologics-switched patients with rheumatoid arthritis: the ANSWER cohort study. Arthritis Res Ther. 2020;22(1):142.

21. Gomes JL, Sepriano A, Eusébio M, et al. Predictors and causes of first-line biologic agent discontinuation in rheumatoid arthritis: data from Reuma pt. Acta Reumatol Port. 2019;44(1):57-64.

22. Narongroeknawin P, Chevaisrakul P, Kasitanon N, et al. Drug survival and reasons for discontinuation of the first biological disease modifying antirheumatic drugs in Thai patients with rheumatoid arthritis: analysis from the Thai Rheumatic Disease Prior Authorization registry. Int $\mathrm{J}$ Rheum Dis. 2018;21(1):170-8.

23. Olsen IC, Lie E, Vasilescu R, Wallenstein G, Strengholt S, Kvien TK. Assessments of the unmet need in the management of patients with rheumatoid arthritis: analyses from the NOR-DMARD registry. Rheumatology. 2019;58(3):481-91.

24. Rashid N, Lin AT, Aranda G Jr, et al. Rates, factors, reasons, and economic impact associated with switching in rheumatoid arthritis patients newly initiated on biologic disease modifying anti-rheumatic drugs in an integrated healthcare system. J Med Econ. 2016;19(6):568-75.

25. Santos-Faria D, Tavares-Costa J, Eusébio M, et al. Tocilizumab and rituximab have similar effectiveness and are both superior to a second tumour necrosis factor inhibitor in rheumatoid arthritis patients who discontinued a first TNF inhibitor. Acta Reumatol Port. 2019;44(2):103-13.

26. Youssef P, Marcal B, Button P, et al. Reasons for biologic and targeted synthetic disease-modifying antirheumatic drug cessation and persistence of second-line treatment in a rheumatoid arthritis dataset. J Rheumatol. 2020;47(8):1174-81. 